\title{
Design of an Improved Ion Chamber for the SNS*
}

\author{
R. L. Witkover† and D. Gassner, \\ Brookhaven National Laboratory, †TechSource, Inc., Santa Fe, NM
}

\begin{abstract}
Ion chambers are in common use as beam loss monitors at many accelerators. A unit designed and used at FNAL and later at BNL was proposed for the SNS. Concerns about the ion collection times and low collection efficiency at high loss rates led to improvements to this unit and the design of an alternate chamber with better characteristics. Prototypes have been tested with pulsed beams. The design and test results for both detectors will be presented.
\end{abstract}

\section{BACKGROUND}

The ion chambers (IC's) designed for the FNAL Tevatron by Shafer in $1982^{1}$ and built by Troy-Onics ${ }^{2}$, have been used at both FNAL and BNL. These are simple in design, consisting of a hollow nickel inner electrode and a nickel foil cylinder outer electrode in a glass enclosure filled with argon. During testing of the chambers for use in RHIC at $\mathrm{BNL}^{3}$ it was found that detector response varied unacceptably using the preferred bias voltage polarity. Normally, from space charge considerations, electrons are collected on the inner electrode. The IC's were tested with a Cs-137 source which produced $1 \mathrm{Rad} / \mathrm{hr}$ at the chamber. The signal current was measured as the bias voltage was varied. The resulting curve is characterized by a rapid increase in signal which quickly rolls over ("knee") into a flat region ("plateau") as the bias becomes high enough collect all charge produced. When the electric field is sufficient to cause multiplication, the chamber enters the proportional region. Results for the first 40-50 RHIC IC's varied widely with many having extended "knees" and early onset of the proportional region. Test results obtained from FNAL for the original detectors did not this show behavior but it was stated that a "scrubbing" process had cured initial problems. Unfortunately, the details of this process are no longer available. It is possible that the original FNAL detectors did not have the same problem since they were built 15 years earlier and subsequent vendor technicians may have unknowingly changed details of the construction. However the detectors were not usable as delivered.

At BNL conditioning using a Tesla coil and "spot-knocking" showed some success but required significant time per unit. It was suggested that the opposite bias polarity would improve uniformity. Tests showed excellent unit-to-unit reproducibility when ions, rather than electrons, were collected on the inner electrode, so it was decided to use the "unconventional" field polarity for RHIC. The major consequence was lower collection efficiency (saturation) for high dose rate losses, and slower signal risetimes, which were not expected to be a problem in RHIC. For SNS, however, this drop in efficiency could be significant for the worst-case $1 \%$ local loss.

*Work performed under the auspices of the U.S. Department of Energy. 


\section{SIGNAL RISE TIME CONSIDERATIONS}

With a 1 msec pulse width, signal risetime is important in the SNS. Electrons are collected in a few microseconds with either bias polarity, but the heavier ions take much longer moving to the inner electrode. At $2000 \mathrm{~V}$ the ion collection time would be close to $700 \mu \mathrm{sec}$. If the losses were constant the signal would continue to rise over the $1 \mathrm{msec}$ Linac pulse as the slow ions arrived, followed by a tail for another 700 $\mu$ sec after the pulse. While the electron signal would allow rapid beam abort for large fast losses, the waveform during the pulse would require unfolding.

Electron and ion charges are generated equally, but will produce equal voltages only for a parallel plate geometry. For cylindrical geometry, using the relative capacitance of a line charge to the 2 electrodes, Shafer ${ }^{4}$ showed that the fraction of current due to electrons at the inner electrode (anode) is:

$$
<F 2>=\frac{2 \pi \int_{b}^{a} F 2(R) R d R}{2 \pi \int_{b}^{a} R d R}=\frac{2 x^{2} \operatorname{Ln}(x)-\left(x^{2}-1\right)}{2\left(x^{2}-1\right) \operatorname{Ln}(x)} \quad \text { where } x=a / b
$$

For the FNAL ion chambers, $a=0.75^{\prime \prime}$ and $b=0.125 "$ and $<F 2>=0.749$. That is, the fast electron signal will be 3 times the slow ion current with the conventional polarity and inverse for the reverse-bias polarity. This was observed in tests in the RHIC transfer line. Clearly, the FNAL chambers would be unsuitable for SNS unless the conventional bias polarity could be used.

The positive ion transit time is also a concern. It is given by:

$$
t=\frac{d^{2}}{\mu_{0} V\left(P_{0} / P\right)}
$$

where:

$$
\begin{aligned}
& \mathrm{d}=\text { Effective electrode separation [cm] for cylindrical geometry } \\
& \qquad d=\left[\left(a^{2}-b^{2}\right) \frac{\ln \left(\frac{a}{b}\right)}{2}\right]^{1 / 2}
\end{aligned}
$$

$\mu_{0}=$ Ion mobility at STP $\left[\mathrm{cm}^{2} /(\mathrm{V}-\mathrm{sec})\right]$

$\mathrm{P}_{0}=$ Atmospheric pressure

$\mathrm{V}=$ Applicd Voltage $[\mathrm{V}]$

$\mathrm{P}=$ Working pressure

The transit time can be reduced by decreasing the electrode gap but with increased gradient corona may occur. A design was proposed ${ }^{6}$ in which the radii were made closer together and the chamber lengthened to keep the same volume. For the original outer electrode radius, $a=0.75^{\prime \prime}$ and inner radius $b=0.5^{\prime \prime}$, at $3 \mathrm{kV}$ bias, the ion 
collection time would be reduced from $560 \mu \mathrm{sec}$ to $72 \mu \mathrm{sec}$. The calculated results are shown in Figure 1. "Negative bias" refers to negative voltage on the outer electrode. Clearly the "positive bias" case is not suitable for SNS. The FNAL chamber with negative bias might be acceptable since the electrons contribute $75 \%$ of the signal within a few microseconds. The new chamber design provides the best response.

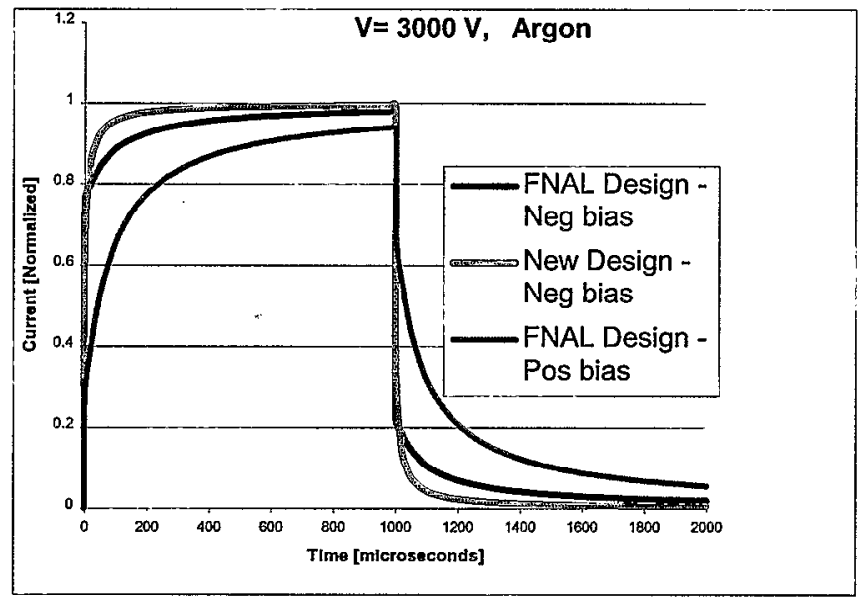

Figure 1. Calculated signal for SNS Linac pulse on original FNAL and new BLM.

\section{COLLECTION EFFICIENCY}

While the equations describing the collection efficiency have been known since Thomson described them in 1899, they have never been explicitly solved. Approximate solutions were obtained for the simpler parallel plate geometry but can be applied to cylindrical if an "equivalent" gap (eq. 3) is used. Boag and Wilson solved the equations with the assumption that the ionization distributions were second and third order polynomials. Their result, which gives a good fit for efficiency $>0.7$ is given by:

where:

$$
f=\frac{1}{1+\frac{\xi^{2}}{6}}
$$

$$
\xi^{2}=\frac{\alpha}{e k_{1} k_{2}} \frac{P}{P_{0}} \frac{d^{4} q}{V^{2}}
$$

$\mathrm{e}$ is the electron charge, $\mathrm{k}_{1}$ is the electron mobility, $\alpha$ is the first Townsend recombination $\quad \mathrm{k}_{2}$ is the ion mobility, coefficient,

$$
q=\frac{I_{\text {signal }}}{v} \times 3 \times 10^{9}
$$

where $I_{\text {signal }}$ is the signal current generated in the ion chamber by the beam loss and 
$\mathrm{v}$ is the active volume of the ion chamber. For the FNAL IC, the sensitivity is 70 $\mathrm{nA} / \mathrm{rad} / \mathrm{sec}$, and the volume is $110 \mathrm{~cm}^{3}$ so for the accepted upper limit of $1 \%$ local loss, the dose rate is $9.2 \mathrm{krad} / \mathrm{sec}$ during the linac pulse.

Consider a chamber with argon gas:

$\alpha=1 \times 10^{6}\left[\mathrm{~cm}^{3} / \mathrm{sec}\right]$

$\mathrm{k}_{1}=1.8\left[\mathrm{~cm}^{2} /(\mathrm{V}-\mathrm{sec})\right]$

$\mathrm{k}_{2}=1.3\left[\mathrm{~cm}^{2} /(\mathrm{V}-\mathrm{sec})\right]$

$$
\begin{aligned}
& \mathrm{V}=3000[\text { Volts] (Negative bias) } \\
& \mathrm{P}=725 \text { [mTorr] }
\end{aligned}
$$

\begin{tabular}{|c|c|c|}
\hline & FNAL & New Design \\
\hline Outer Radius [cm] & 1.905 & 1.905 \\
\hline Inner Radius $[\mathrm{cm}]$ & .3175 & 1.27 \\
\hline Active Length [cm] & 10 & 17.4 \\
\hline Volume $\left[\mathrm{cm}^{3}\right]$ & 110.84 & 110.21 \\
\hline Effective Gap [cm] & 1.991 & 0.6436 \\
\hline Ionization Density $\left[\mathrm{esu}^{\mathrm{cm}} \mathrm{cm}^{3}-\mathrm{sec}\right]$ & 16850 & 16850 \\
\hline Ion Transit Time $[\mu \mathrm{sec}]$ & 564 & 72.2 \\
\hline Electron Fraction (neg on outer) & .7495 & .5669 \\
\hline Collection Efficiency & .357 & .971 \\
\hline
\end{tabular}

Then

While the efficiency calculation for values $<0.7$ may not be accurate, clearly the new chamber would be superior to the original, both in ion transit time and collection efficiency, but, since the electrode gap is smaller, great care must be taken with the high voltage design. Guard electrodes would be required to decouple the signal electrode from leakage. The design would be more difficult to manufacture than the simple FNAL detector, with higher detector cost. Note that the electron fraction is 0.567 , with $43 \%$ of the signal coming from the now ions.

\section{THE NEW ION CHAMBER DESIGN}

The design of the new ion chamber is shown in Figure 2. Voltage gradients have been reduced by rounding the electrode ends. Guard electrodes divert leakage from the $\mathrm{HV}$ electrode to ground and assure the signal collector is in a uniform electric field region and. They also prevent ions at the chamber ends from contributing to the signal resulting in longer transit times. Three prototypes units have been made. The first was limited to $3 \mathrm{kV}$ when filled with argon due to HV feedthrough breakdown, but when filled with nitrogen was able to reach $5 \mathrm{kV}$. It was then installed in the transport line between BNL Linac and Bonster. Prototype units 2 and 3 were built with a higher voltage feedthrough and improved ceramic design. The radius of curvature of the ends of the guard rings and signal electrodes were also increased. These units have been tested to $4.5 \mathrm{kV}$ with argon. Prototype 2 has been installed in the BNL Booster extraction line for tests with short pulse beams similar to SNS Ring extraction. 


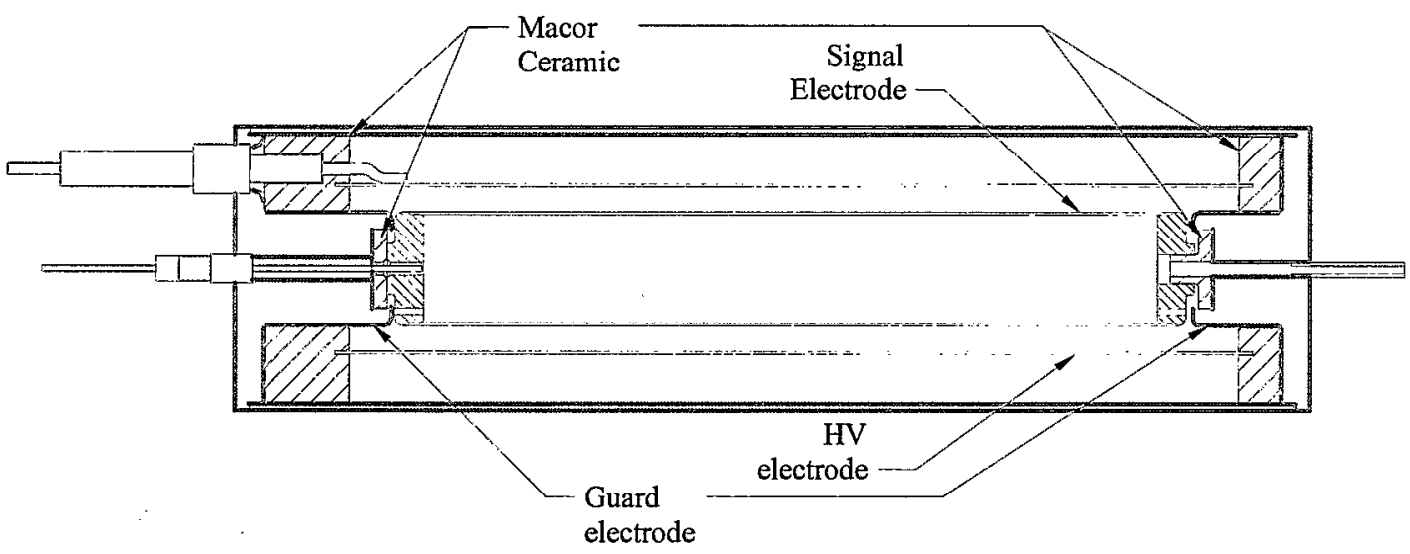

Figure 2. Drawing of new design BLM prototype.

\section{IMPROVEMENTS TO THE FNAL DETECTOR}

As an alternative to the new development, the original was studied to see if modifications could be made to improve its performance. The difficulties with the conventional (negative) bias polarity in the FNAL IC were believed to be due to high field points at the tip of the inner clectrode and at the supports for the outcr clectrode, since these are where breakdown occurs when the voltage is raised. Changes were made to the outer electrode to eliminate crimping at the flared end of the outer electrode at the support pin attachment locations. Figure 3a shows the original outer electrode crimped inward by the support rods. Figure $3 b$ shows a modified chamber with the outer electrode slotted to allow the support to pass the flared end without bending it inward.

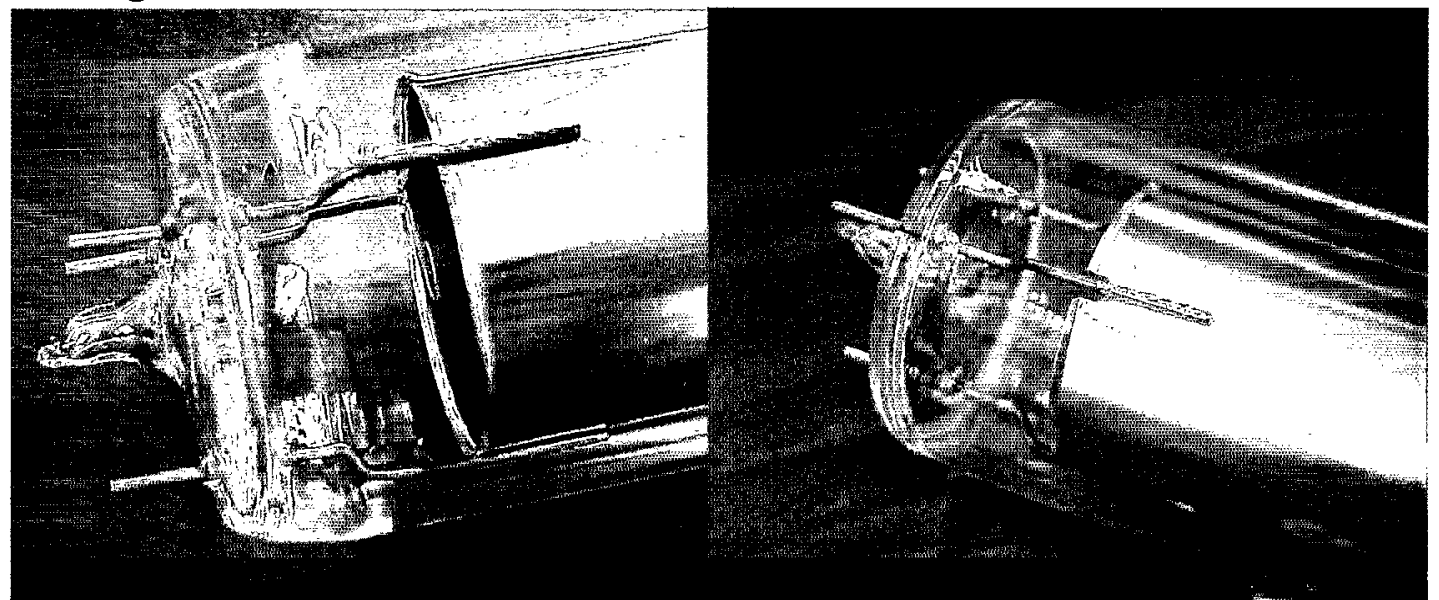

Figure 3a. Original FNAL Outer electrode Figure 3b. Slotted outer electrode

So far 5 chambers have been modified in this way. Tests indicate that this has significantly improved the unit to unit response. Figure 4a shows the cesium source tests of 5 of the first RHIC chambers with conventional bias polarity. Figure $4 \mathrm{~b}$ shows results for 5 units with modified (slotted) outer electrodes. The uniformity is clearly improved with the slotted electrodes. 
It was felt that some of the voltage limitation may have been due to sharp edges at the open end of the hollow center electrode. Several chambers were built with the slotted outer and a rounded solid tip inserted in the end of the electrode. Tests of these units did not show a significant change from the slotted outer electrode. While these modifications seem to have improved the production uniformity, the ion transit time and high dose rate saturation are still a problem for SNS. If the design could be improved to allow higher operating voltage then the ion transit time would decrease and collection efficiency increase. The improvement is somewhat less than linear with voltage, so this might not be very productive.

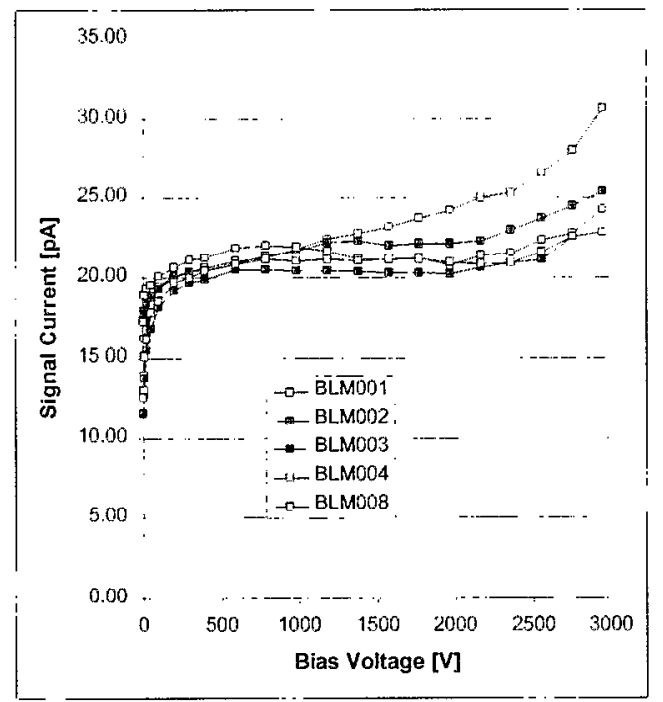

Figure 3a. Original FNAL IC test data

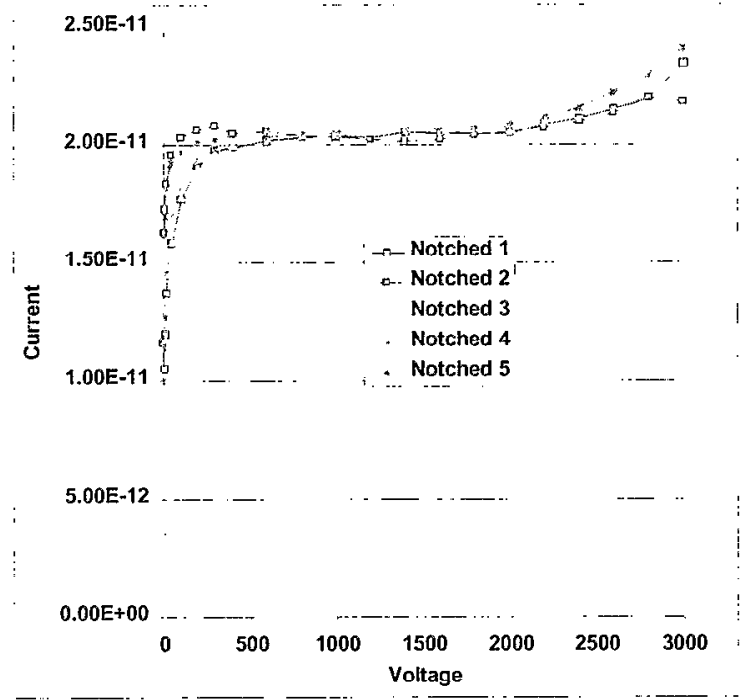

Figure 3b. Modified (slotted) FNAL data

\section{TESTS WITH BEAM}

A non-modified FNAL IC and Prototype 1 were installed together in the BNL Linac-to-Booster (LTB) line, approximated 1 foot from the beam line, where high dose rate losses from the $200 \mathrm{MeV} \mathrm{H}$-minus beam could be observed. Two sets of data runs were made. In the first a high sensitivity multi-channel integrator module of the type used in the source testing of the chambers was used to condition the signals. They were modified to have a $470 \mathrm{Ohm}$ input so rise times of several $\mu \mathrm{sec}$ could be observed on a LeCroy digital scope. The bias voltage was varied to determine the collection efficiency curves for both bias polarities. The beam current waveform was also captured. Figure 5 shows the results of this run. Clearly the positive bias (nonpreferred) FNAL response shows low efficiency, especially at lower bias voltage. The data for the negative bias shows large excursions, while summation of the beam current data does not show more than a few percent variation between data points, implying beam motion. The data for Prototype 1 appears fairly flat throughout the entire voltage scan. Calculations indicate that the FNAL chamber should be $77 \%$ efficient while Prototype 1 should be $92 \%$ at $3 \mathrm{kV}$ for negative bias at this loss level. Dedicated runs are planned to study the efficiency at various dose rate levels. 


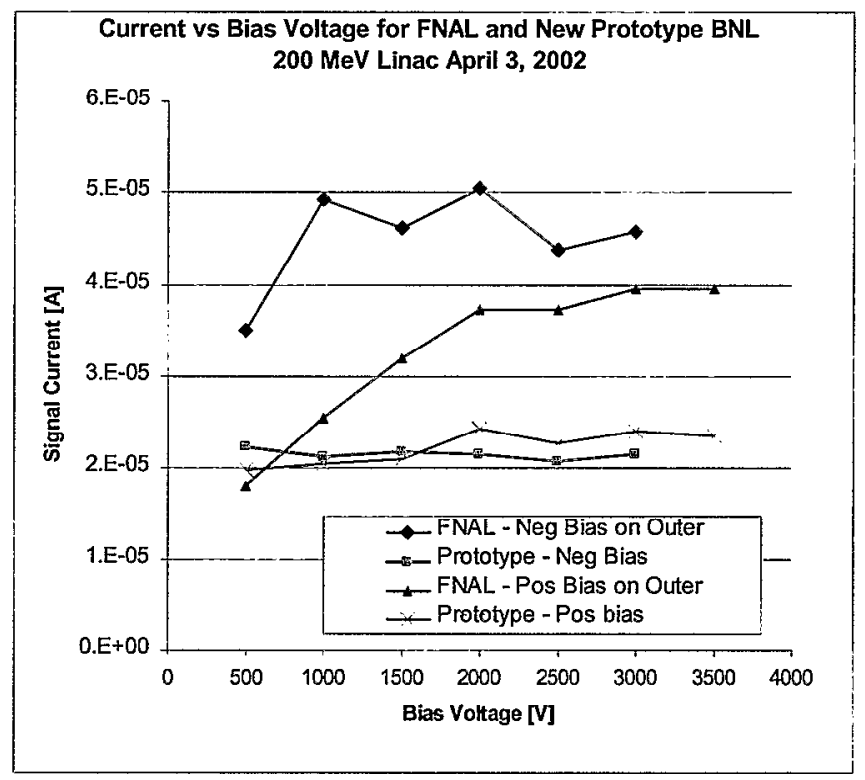

Figure 5. Measured bias curve (collection efficiency) for $200 \mathrm{MeV}$ Linac beam. Aproximately $1000 \mathrm{~W} / \mathrm{m}$ loss.

A second run was made using the prototype analog front end (AFE) circuitry designed for $\mathrm{SNS}^{8}$. This circuit has a current amplifier first stage using a Burr-Brown OPA627BM opamp with outputs from a unity gain (50 kHz BW) buffer, a "leaky integrator" and a filtered $(1 \mathrm{kHz})$ slow amplifier. Data from the fast output and the beam current transformer is shown in Figures $6 a$ and $6 b$.

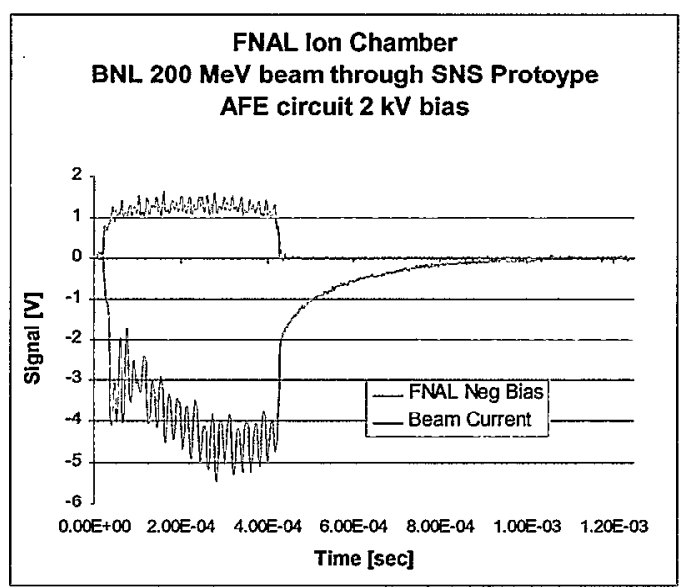

Figure 6a. FNAL IC Beam Test.

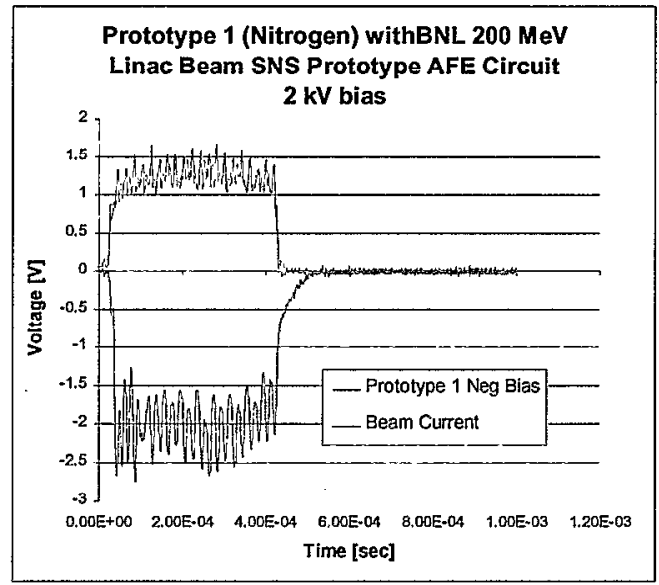

Figure 6b. Prototype 1 IC beam test

The signal from the FNAL chamber (Figure 6a) can be seen to have a very long tail following the pulse, due to the slower ions being collected after the beam. They are also arriving during the pulse, causing the gradual rise rather than directly following the beam current (also see Figure 1). The apparent noise during the pulse is actually modulation of the beam current to tailor the stacking in the Booster. The signal from the Prototype ion chamber has a much faster ion collection time, as expected. 


\section{CONCLUSIONS}

Improvements have been made to the original FNAL detector design which result in more uniform characteristics of the production units. These changes allow the use of the conventional bias polarity (center electrode is anode) without conditioning of the chambers and further development may result in moderately increased operating voltages in the future. However, concern over collection efficiency (linearity) at high dose rates may preclude its use in SNS. The slow ion transit times are comparable to the SNS pulse width, requiring unfolding to obtain the true time behavior.

The new chamber design appears to yield significantly faster ion transit time and higher collection efficiency at high dose rate, as predicted. However, higher fabrication costs and the uncertainties of bringing a new design to production need to be weighed against the benefits to the SNS application. A firm specification of linearity at high dose rate is needed before the detector choice can be made.

\section{ACKNOWLEDGMENTS}

The authors wish to acknowledge the contributions of C. J. Liaw in the mechanical design, Dave Kipp is assembling the prototype chambers and Paul Ziminski in testing the chambers with the cesium source. The prototype circuit used in the beam tests was built and tested by Chaofeng Mi.

\section{REFERENCES}

\footnotetext{
${ }^{1}$ R. E. Shafer, et al., The Tevatron Beam Position and Beam Loss Monitoring Systems", Proc. $12^{\text {th }}$ Int'1 Conf on High Energy Accel., FNAL (1983) p609

${ }^{2}$ Troy-Ionics, Inc., Kenvil. NJ

${ }^{3}$ Witkover, R. L., Michnoff, R. J. and Geller, J. M., "RHIC Beam Loss Monitor System Initial Operation", Proceedings of the 1999 PAC, NY, 1999, 2247

${ }^{4}$ Shafer, R. E., Private communication

${ }^{5}$ Boag, J. W. in "Radiation Dosimetry, Vol. Il", F. Attix, et al, eds.,Academic Press (1966), p22

${ }^{6}$ R. Witkover," Proposal for a Quasi-Parallel Plate, Cylindrical Geometry Ion Chamber for SNS", November 7,2001, unpublished

${ }^{7}$ Boag, J. W. and Wilson, T., "The saturation curve at high radiation intensity". Brit. J. Phys., 3 222-9. (1952) and Loc. Cit (5), p16

${ }^{8}$ Witkover, R. L. and Gassner, D., "Preliminary Design of the SNS Beam Loss Monitoring System". To be published in these proceedings.
} 